\title{
Medical Information Sharing Applying Blockchain Technology
}

\author{
Sang Young Lee \\ ${ }^{1}$ Professor, Namseoul University, South Korea \\ ${ }^{1}$ sylee@nsu.ac.kr
}

\begin{abstract}
In this paper, we proposed a method of medical information blockchain technology to the medical field and using it for PHR applications. Here we propose a structure and technology for various applications of hospital data for remote control and sharing of various PHR data for the efficiency of medical information. Blockchain technology is a distributed database, which is a ledger technology that records changes, for example, adding information, revising, and deleting within the unit of blocks and share. The form used here means a list derived from medical practice, but the shared information applied with blockchain technology can be applied to all data. And blockchain technology is applied to PHR service to transmit and store medical information to medical institutions that use existing information to utilize medical information. In addition, medical information can affect distributed databases that provide storage and backup/restore functions. Through this, the server for the blockchain of each participating institution of the blockchain network can serve as an interface for the utilization of medical information. Blockchain also manages access, processing, and disposal regarding medical information, providing verification and inspection of medical information along with maintaining the integrity of medical information and satisfying the previously recorded security requirements.
\end{abstract}

Keywords: Blockchain, medical, information, sharing. 


\section{Introduction}

As interest in the medical environment is increasing, the demand for medical services is also growing. Therefore, the establishment of a foundation for the development of medical services became the center of attention and the medical information sharing business has started. Since medical information system has been converted into EMR, health information has created and distributed by nonmedical personnel because of interest in health care is increasing and the expansion of smart device (Information Security Reference Model for u-Health Service, 2010).

If health information recorded by individuals is applied to medical research in an unproven state, it may cause wrong results, therefore it is impossible to use it directly in the medical site. The unreliable medical information may be injected into medical research and development due to malicious attacks from inside and outside.

The construction of medical infrastructure that connects and uses information in the current medical information system is the most important effort of the medical industry. This goal is to first enable services for various users in the HIS environment. In other words, it was realized thanks to the efforts of the standards organization that established standards in the medical field. Users establish data model standards with an emphasis on operability when using data in medical institutions. Standards bodies set standards for terms that give these data precise meanings. A variety of healthcare organizations utilize workflow standards to support processes for patient care. Finally, various institutions achieve the provision of medical interoperability standards that have been given interoperability with actual guideline standards and standardized organizations.

Medical information is a study that studies the interface between medical care and computer technology, and studies various complex fields. In HIS, it is a technology that interacts with components with different environments for various technologies in the medical field. An important technique for these HISs is interoperability. HIMSS defines HIS as "health information systems that work together within and outside organizational boundaries for personalized and effective health care delivery". And HIS applications make the interoperability testing process of medical information systems difficult. This aspect is the subject of research so far.

Interoperability tests of various HIS are performed by connecting various systems. An example of such an interoperability test event is a user connection event. However, there are many shortcomings in practically performing tests that connect and check various systems so far. These techniques apply to all kinds of systems, regardless of which institution they belong to. It starts from the basic that various systems are interconnected and operated.

And HIS constitutes the information system of various medical institutions and is the entire medical field system including medical education, research, etc. as well as 
medical care. This test-based approach is aimed at practical use. Figure 1 shows examples of the various interactions between the introduced application roles of these three HIS systems. HIS systems functionally describe different types of users. Each system serves several top-level applications. A transactional system placing an order requires the entire operation in the direction of the hypothetical system. And it transmits this information to the system to run the whole application program.

In related fields, blockchain technology is a digital ledger technology that can safely keep an ever-growing list of data records and transactions. Blockchain is the driving force behind potentially transforming healthcare (Kratz et al., 1999). It simplifies and accelerates the way data is processed in areas such as revenue cycle management, health data interoperability and supply chain validation in a variety of health-related industries. Blockchain reduces data entry by users. It can dramatically reduce the resulting maintenance costs and improve data accuracy and security (Kratz et al., 1999; National Electrical Manufacturers Association, 2015).

Blockchain is centrally managed based on medical information. Healthcare organizations are using multiple technologies to make secure and reliable transactions for interacting systems with less reliance on other systems. In other words, blockchain is a platform that supports transactions. These transactions provide decentralization, centralization and pathways through cryptography and game theory. The technology offers technology across multiple application domains, including cryptocurrencies and decentralized technologies.

Blockchain is based on smart contracts. The Ethereum blockchain is an improvement on the implemented blockchain technology. That is, the exchange of digital assets, such as cryptographic tokens, or some data, is achieved between two or more parties according to specific rules (Zhang et al., 2018; Dierendonck and Arbesser-Rastburg, 2004).

Various medical institutions apply medical contracts to build safe and effective technology infrastructure. In other words, better information about healthcare coordination can lead to improvements for individuals and communities. In the future, interoperable software and platforms in healthcare environments of healthcare data will securely communicate and exchange data between healthcare organizations and technology providers. The data exchanged here should be available. These health information systems coordinate secure access to electronic health records (EHRs), enabling health care providers to collaborate inside and outside organizational boundaries (Zhang et al., 2018). And to achieve the integration/medical provision of information for individuals and institutions.

Generally, Blockchain technology is a distributed database, which is a ledger technology that records about changes, for example, adding information, revising, and deleting in the unit of blocks and share. The medical information used here basically means the transaction content, but blockchain technology can be applied to all data as safely shared information (Dierendonck and Arbesser-Rastburg, 2004). 
In general, security becomes a great concern when using cloud computing.
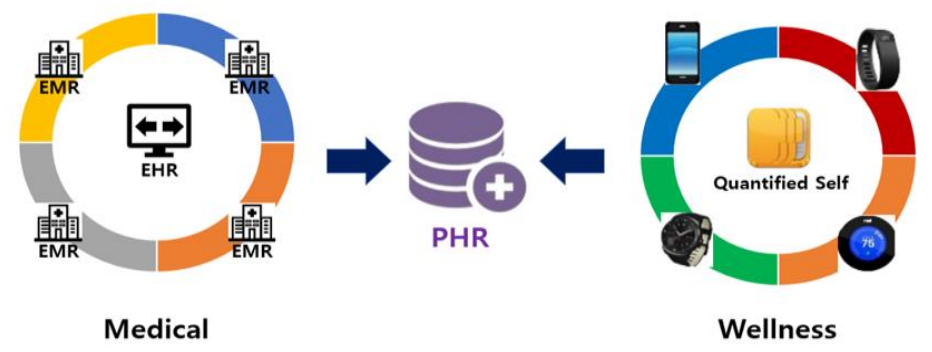

Especially medical institutions use a remote cloud base to manage and protect sensitive and confidential data related to personal medical care on basis of cloud services. Therefore, the stability of companies that manage hospital data is very important in terms of security. Not only that, patients may feel uncomfortable giving their medical data to third parties. Which means medical institutions are obliged to protect data from unauthorized users by selecting a reliable cloud service provider. The verification of security and proven performance analysis is more important than efficiency in a cloud computing environment (Zhang et al., 2018; Dierendonck and Arbesser-Rastburg, 2004).

Fig. 1: Structures of PHR

The medical information platform is implemented through PHR in the central management aspect. Patients in medical institutions can search and share medical information. In addition, the patient can use the computer at home to manage and utilize drug information, insurance-related information, prescription and medical records, etc. And it can be used for user's PHR utilization, PHR update through medical institutions, drug interaction check, doctor/hospital search, etc. (Pelgrum et al., 2011; Gunawardena et al., 2008) .

Basically, PHR targets all personal health-related information used by hospitals. That is, it includes the overall service/information/platform for personal health.

EMR is a medical information system created and used within a single medical institution, and EHR is a medical information system that complies with national standards for interoperability and used by multiple medical institutions. The personal medical information saved in the EMR and EHR becomes the important basis of PHR. And PHR has developed centered on various institutions. In addition, various systems and managed personal health information can be easily checked and integrated. These systems can be shared with each other (Peng and Morton, 2010; Vikram, 2011; Humphreys et al., 2005).

It is also developing into a model that provides useful services such as self-health management, disease prevention, disease prevention related to medical institutions and insurance companies, and follow-up management. In the future, these systems 
will add not only genetic information but also specialized information such as social networking and behavioral habits to analyze new health indicators and provide customized disease prevention health care. In this future, blockchain technology is applied in the medical field. Based on the PHR, it provides a way to use it in your application. Use medical data for easy and secure control and sharing of efficient PHR data. Blockchain provided an architecture for gateway applications of medical information.

\section{Related Works}

Medical information that was record by hand of medical personnel in the past has been converted to EMR (Electronic Medical Record) with the introduction of an information system. As interest in personal health increased, the concept of medical information is changed into EHR (Electronic Health Record), and recently, its scope has expanded to PHR (Personal Health Record) with the spread of Wearable Devices, IoT, and Smart Devices (Peng and Morton, 2010; Vikram, 2011). As the number of the population increased, medical personnel and the amount of medical information is increased naturally. The goal of the medical information system is to increase the utilization of medical information, which is increasing significantly. In this goal, the data quality of medical information is important. Therefore, international standards for medical information in related fields are used (Humphreys et al., 2005; Xu and Morton, 2018).

In the current medical field, researchers and users experience fragmentation and isolation of information. It also causes systemic disruption due to delayed communication and disparate workflow tools. And data controllers attach great importance to patient health and identifiable information safety maintenance regulations. Thus, the perception of preventing information sharing (including anonymization) and the makes data exchange difficult. In addition, medical institutions have medical systems that are difficult to be compatible with each supplier. It creates an information gap in medical communication, making it difficult to manage and deliver patient privacy-centric care (Rino, 2011; Lee, 2021).

We followed a four-step valuation methodology that we previously developed technologies to evaluate PHR in various healthcare organizations. An efficient fourstep process is as follows (Sekhar and Suneetha, 2018; Kim et al., 2018).

- Medical technology definition and data collection

- Standard term classification definition and implementation framework

- Synthesis of implementation information

- New model development

1. Medical technology definition and data collection. There are many definitions of various PHRs, but they use a generic PHR function. 
A personal health record (PHR) is information infrastructure that allows patients to access and coordinate lifelong health information and make the appropriate pieces of information available to those in need (Pelgrum et al., 2011; Gunawardena et al., 2008).

2. Standard Terminology Classification Definition and Implementation Framework. PHR analyzes and organizes the value of information. Developed a PHR classification and evidence framework.

The PHR classification used classifies the PHR function according to the information of the medical institution and classifies the information from the patient's point of view. It contains both healthcare infrastructure and application components.

As seen in other studies, within the PHR framework, we envisioned four PHR architectures based on the primary data sources for PHR. This includes provider information, payer information, third parties and interoperable PHRs. A single intelligence agency includes both shared supplier-information and payerinformation and third-party PHRs. Currently, interoperable PHR is leveraged based on strong standards for electronic medical data exchange.

3. Synthesis of implementation information. Ultimately, various PHR frameworks organize and integrate data from the domains and experts they use. In other words, this PHR enhances the value of information. The embodied information constitutes a value cluster (the general area a PHR has or may have value). It has a PHR function with potential value in this value cluster. PHR demonstrates the potential effectiveness of various infrastructure and application functions for administrative information and clinical purposes. The model used here utilizes various PHR functions.

4. Developing new models: Healthcare organizations incorporate evidence to reduce costs and increase profits. Information develops a computer model that estimates at a global level. We use expert opinion and relevant evidence from nonPHR sources as there is relatively little quantitative evidence of PHR costs and benefits through literature review. In these literatures, derived model parameters were used. Our model consists of a profit model using the overall PHR and a PHR cost model. Combined to assess the net worth of PHR.

The overall benefit of using PHR is that it enhances the proven value of a very small number of existing PHR functions, although there may be many PHR functions in future PHRs due to modeling.

Now it became possible to exchange and share medical information among various institutions such as hospitals, medical research institutions, and legal institutions. As interest in such a medical environment increases, the demand for the development of medical services is soaring. Therefore, the establishment of a foundation for the development of medical services is emphasized and various studies on medical information have been conducted (Mallik and Neeraja, 2019). 
These studies comply with international medical system which generates medical information through medical information systems such as OCS, EMR, and EDI. The standard is applied to the information but the method of saving the information vary depending on medical information system.

To properly use medical information, an appropriate interface is required which is converted into format of various medical information system that can be retrieved and inquired, and medical information can be transmitted to various medical institutions. Out of the various technologies and methods, the most effectively proposed method is Registry (Metadata) Server and Medical Gateway. The representative use of Blockchain in the health site is electronic medical records. And medical information is divided into EMR centered on electronic records, EHR centered on mutual exchange, and PHR centered on personal health records.

Research in the field of related medical information aims to manage personal data for creating and storing medical-related electronic documents and management of health-related factors. In fact, studies on EMR use cases of Blockchain have mainly been conducted on Blockchain decentralization, immutability, data sources, reliability, rigidity, contracts, security, and privacy. This study focuses on the use of blockchain for storage and management of patients' electronic medical records (EMR) and how to facilitate patient-centered data sharing in various medical services.

A blockchain is a list of blocks connected by multiple chains. These block lists are based on data structures. A typical blockchain is managed over a private network with up-to-date versions of all data. The blocks used are recorded by the user who utilizes the data. A blockchain based on Bitcoin serves as a "repository of solid, immutable and reliable information." Here, it is one of the distributed ledger technologies, which is a distributed, shared and encrypted database of information. A monolithic blockchain consists of interconnected chains and is a complete data structure in its format (Xu and Morton, 2018; Rino, 2011).

- Blockchain version: Indicates the validation rules based on the version block.

- Upper block code: Consists of 256-bit hash rate.

- Merkle Tree Root Hash: The hash of the transaction.

- Block Timestamp: Shows the current value per second.

- nBits: Format as a simple hash.

- Nonce: An incrementing 4-byte field. 


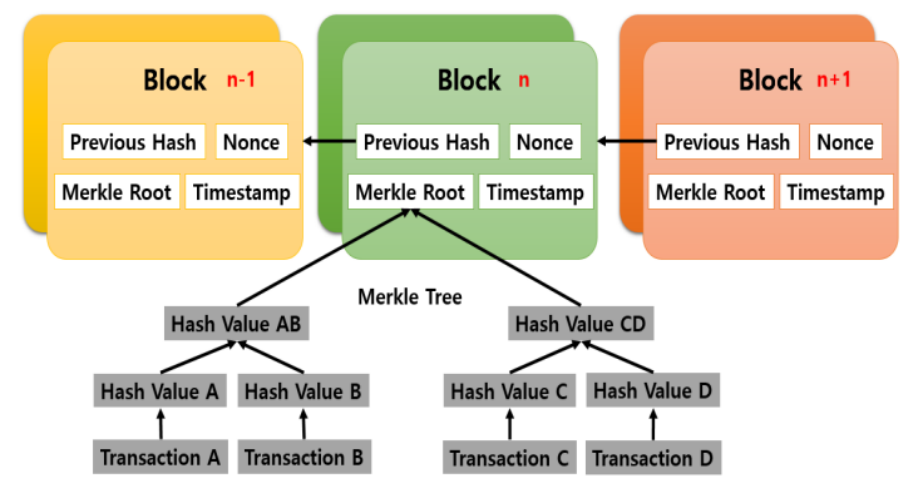

Fig. 2: Structures and Elements of Blockchain

Figure 2 shows structures and elements of blockchain. Especially, research has been conducted on technology and data sharing, processing or use in order to build a medical platform that patients can control their own methods. Applications using Blockchain technology for healthcare data management are introduced to apply perfect security technology in medical system design. This study proposes an architecture for a gateway application of healthcare data for easy and safe control and sharing. In other words, Blockchain technology uses multi-step authentication that can protect each different medical data of patients. And, for the security of medical data, a research proposes scenario related to biometrics and biomedicine.

Especially, research has been conducted on technology and data sharing, processing or use to build a medical platform that patients can control their own methods. Applications using Blockchain technology for healthcare data management are introduced to apply perfect security technology in medical system design. This study proposes a gateway of medical data for functionally secure sharing.

And, for the security of medical data, a research proposes scenario related to biometrics and biomedicine. A blockchain is a list of blocks connected by multiple chains. These block lists are based on data structures. A typical blockchain is managed over a private network with up-to-date versions of all data. The blocks used are recorded by the user who utilizes the data. A blockchain based on Bitcoin serves as a "repository of solid, immutable and reliable information." Here, it is one of the distributed ledger technologies, which is a distributed, shared and encrypted database of information. A monolithic blockchain consists of interconnected chains and is a complete data structure in its format ( $\mathrm{Xu}$ and Morton, 2018; Rino, 2011; Lee, 2020). 


\section{Medical Information Sharing}

Personal Health Record (PHR) has been introduced as a patient-centered health information model. PHR services in medical institutions can efficiently create, manage and control personal patient information. Which means they can manage data through efficient tools for saving, retrieving, and sharing medical information.

Various PHR systems can manage individual patient information to manage their own medical records and share them with various users.

In recent years, sleep motoring device or smart home devices have been introduced, and it is expected that loT personals will actively discuss PHR-related health care standard in the future. PHR can manage data centered on patients. In other words, it is an application that patients use.

The goal of various PHRs is to enable individual patients to safely and conveniently collect and manage health information. Hospital information providers also manage hospital visit data, immunization records, prescription records, and physical activity data collected through various channels. Individual patients can control how their health information is used and shared through PHR.

However, it faces obstacles similar to the existing heterogeneous EHR systems because this approach does not solve the core data sharing problem. Blockchain, however, allows individuals to control through dispersion. We use algorithm that various participants agreed. It ensures that participants can widely access their information and data distribution is guaranteed. Through a service connected to the existing health system, The PHR has the following advantages.

o data access control

o Know the source of the collected data.

o Notify when a provider accesses data.

o It is always transparent to the patient through the data log.

o Patients can search health information anytime, anywhere.

People who need specific information can use. Medical service users can make better decisions, access information necessary for treatment, and effectively communicate between patients and medical personals through PHR.

The order of sharing medical information based on Blockchain is as follows. Creating the Block, transmission the Block, receiving block verification, and saving the Block for the medical information to be shared. In the case of applying Blockchain to a specific information service, the subject which created or changed information makes the Block the information to be shared. It is transmitted to all nodes participated in the Blockchain Network. And the node on the Blockchain Network verifies the transmitted information block.

After that, a normal block shares information transmitted by senders in the way of registering and saving in the Chain. Chain information saved in a specific node on the Blockchain Network can be forged by an attack of an unauthorized person. 
However, Blockchain Network applies the principles of PoS, where forged Blocks or Chains in one Node are rejected. All nodes must be simultaneously forged but this is practically impossible.

Blockchain technology used by medical institutions can satisfy the integrity of shared information through technical mechanisms. We are constantly adding methods of blockchain modding without deleting or modifying the blocks we use. With such blockchain technology, information can be tracked only with chain management technology, without the need to implement unnecessary versioning.

These advantages of blockchain are being applied and expanded to information services that require authentication/verification of shared information.

\section{Architecture With Blockchain Platform}

As medical information includes personal information, there are many kinds of security requirements, requirements to ensure safety of data, not only the patient's records. Basically, reliability, integrity, and traceability should be guaranteed along

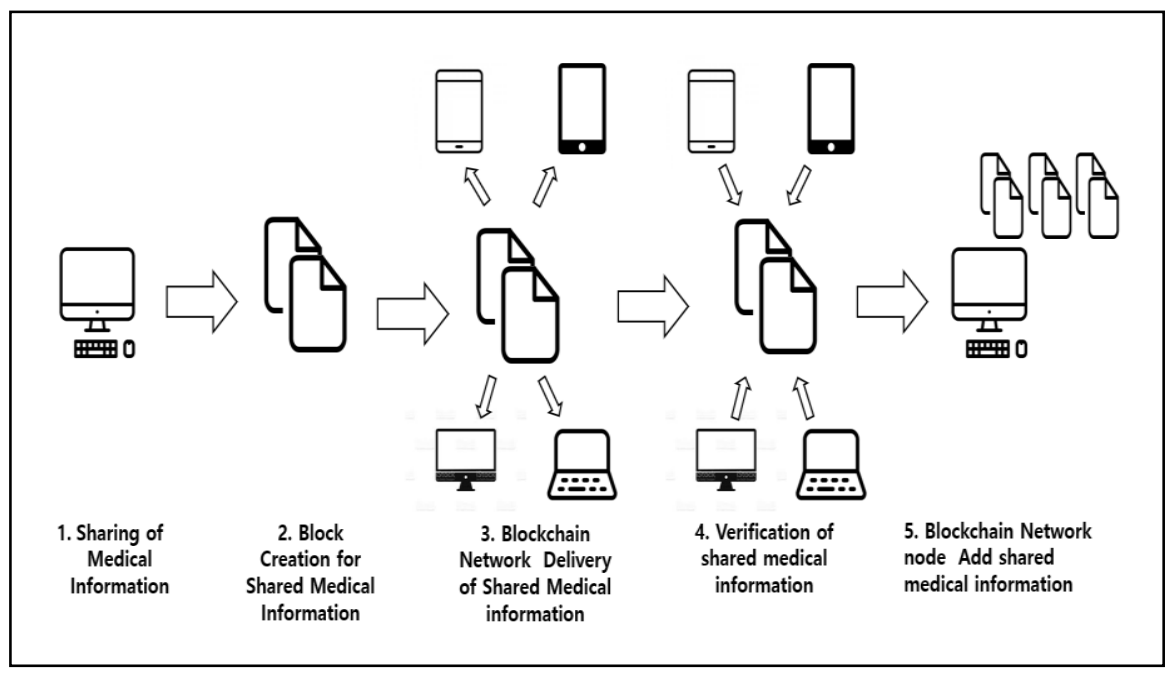

Fig. 3: Medical Information Sharing

with confidentiality to prevent leakage of personal information.Currently, HL7 and DICOM present security requirements as shown in the table below. It is a security measures standard for each layer of medical information.The security threats and security requirements for medical information are as follows. Shortly, medical information should have the reliability of medical information for the entire lifecycle of its creation, saving, transmission, and shredding.

And medical information requires comprehensive security features such as user certification, rights to management, confidentiality, integrity, traceability, and nonrepudiation. A medical information sharing service that is used in various fields 
should satisfy all of the previously identified security requirements as a basis for medical service development. This study suggests Private Blockchain including Life-Cycle of medical information sharing technology and the research and efforts.

Table 1: Security Requirement of Medical Information Standard

\begin{tabular}{|c|c|c|c|c|}
\hline Security Requirements & HL7 & DICOM & $\begin{array}{c}\text { TTAK.KO } \\
\mathbf{- 1 0 . 0 3 0 4}\end{array}$ & HIPAA \\
\hline Authentication & o & o & o & o \\
\hline Authorization & o & & 0 & 0 \\
\hline Data Confidentiality & o & o & o & o \\
\hline Audit & o & & o & o \\
\hline Secure Communication & o & & 0 & o \\
\hline Integrity & o & o & o & o \\
\hline Key Management & & o & & \\
\hline
\end{tabular}

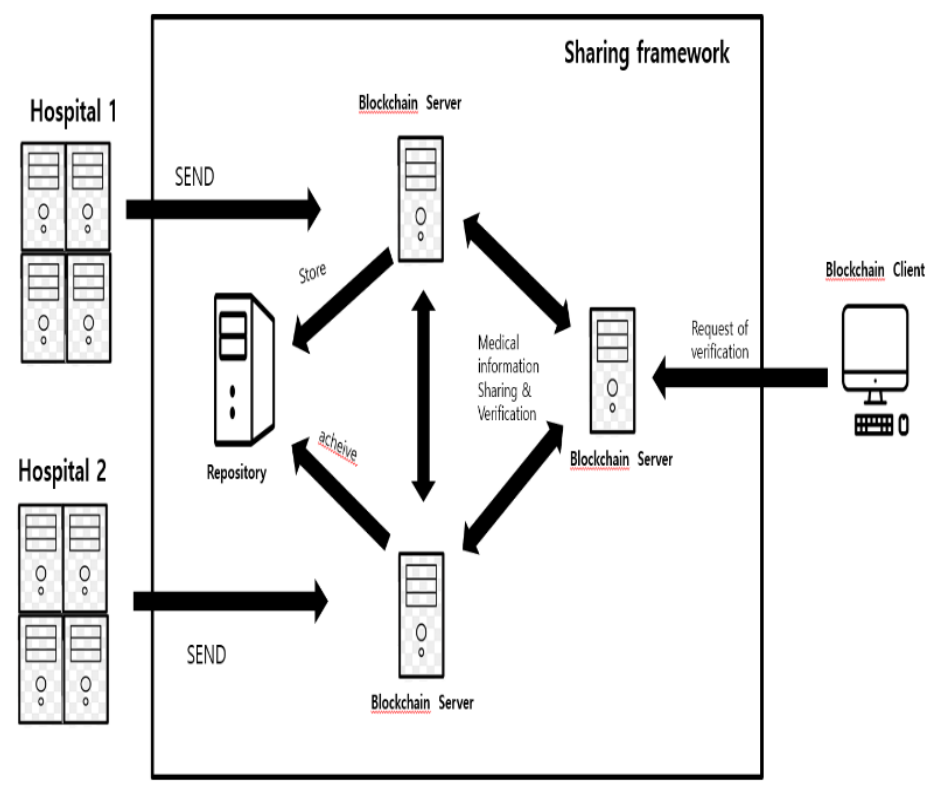

Fig. 4: Blockchain-based medical information sharing service

Blockchain technology is applied after expanding the current medical information collection service to medical information sharing services by area. The components of this mechanism are as follows.

It consists of a Blockchain Client that creates or utilizes medical information to be shared with the Blockchain Network which is consisted of the Blockchain Server.

First, medical information created by individual medical institutions is made as a 
block and then transmitted to the Blockchain Network.

For the use and verification of medical information, the Blockchain Client approaches the Blockchain Server to search or request for verification. Upon receipt of a request, Blockchain Server transmits or verifies medical information according to the request.

The Blockchain-based framework is as follows. In order to share medical information, Data Cleansing has to be applied, but in terms of medical information, Data Customizing is required for each institution's features. Therefore, for the sharing and use of medical information, there should be a proper conversion of medical information. This study applies a medical information sharing service that combines medical information sharing service and blockchain technology.

The Blockchain Client and Server, that are participating nodes for each medical information sharing service, both apply a layer for data handling.

First, the sharing of medical information created by each medical institution is converted into a Block by the Blockchain and transmitted to the Blockchain Server. After that, the Blockchain Server receives it and verified by the Blockchain. The verified medical information contains the adjusted information, and the medical standard. To register the medical information to be shared in the Repository, the Mediator converts it into a standard shared format.

The converted medical information is separated from the indicator into actual medical information and the metadata is contained in the register while the actual medical information is saved in the Repository. The access process for the use of medical information proceeds in the reverse order of the sharing process. If a medical institution wants to use a medical information, they can use it through the RIF.

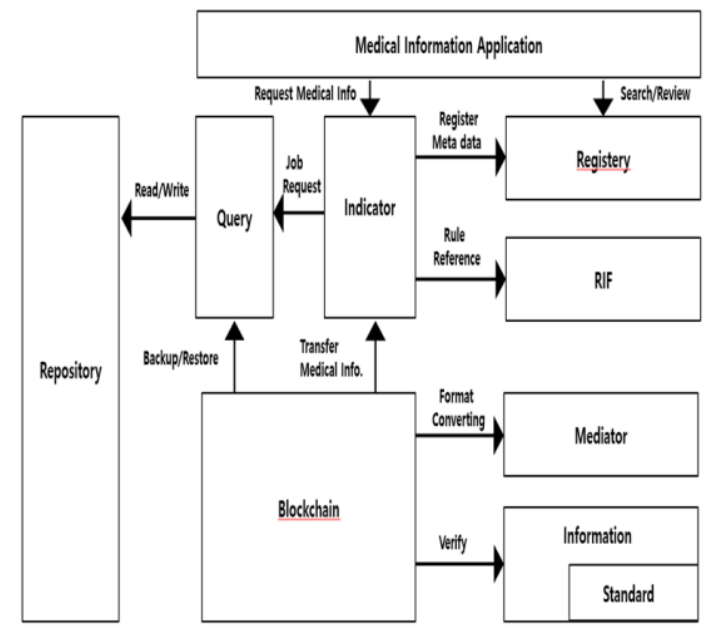

Fig. 5: Medical information sharing service 


\section{Conclusions}

In this paper, we proposed a method of medical information blockchain technology to the medical field and using it for PHR applications. Here we propose a structure and technology for various applications of hospital data for remote control and sharing of various PHR data for the efficiency of medical information.

Blockchain technology is a distributed database, which is a ledger technology that records changes, for example, adding information, revising, and deleting within the unit of blocks and share. The form used here means a list derived from medical practice, but the shared information applied with blockchain technology can be applied to all data. And blockchain technology is applied to PHR service to transmit and store medical information to medical institutions that use existing information to utilize medical information.

The chain also manages access, processing and disposal regarding medical information, providing verification and inspection of medical information along with maintaining the integrity of medical information and satisfying the previously recorded security requirements.

It should not be overlooked that Blockchain technology itself does not provide confidentiality. This requires encryption saving in the chain block, applying IPsecVPN(Virtual Private Network) in the transmitting process and applying encryption channel SSL(Secure Socket Layer) to protect medical information from leakage that is not authorized.

Furthermore, the medical information itself is very important, therefore a private blockchain network has to be applied, and for this, a Server or Client management on the blockchain network, identification and authentication for each node, and authority management must also be applied.

\section{Acknowledgments}

Funding for this paper was provided by Namseoul University.

\section{References}

Dierendonck, V. A. J., \& Arbesser-Rastburg, B. (2004). Measuring Ionospheric Scintillation in the Equatorial Region Over Africa, Including Measurements From SBAS Geostationary Satellite Signals. Proc. ION GNSS.

Gunawardena, S., Zhu, Z., \& van Graas, F. (2008). Triple Frequency RF FrontEnd for GNSS Instrumentation Receiver Applications. Proc. ION GNSS, Savana, GA. 
Humphreys, T., Psiaki, M., Kintner, P., \& Ledvina, B. (2005). GPS carrier tracking loop performance in the presence of ionospheric scintillations, ION GNSS Long Beach CA, 156-167.

Information Security Reference Model for u-Health Service, (2010). Information Security Reference Model for u-Health Service, TTA Standards.

Kim, H. G., Park, J. T., \& Moon, I. Y. (2018). Research for Applying Big Data System to Internet of Things devices using Web Technology. International Journal of Mobile Device Engineering. 2(2).

Kratz, M., Humenn, P., Tucker, M. Nolte, M., Wagner, S. Seppala, G., Shadrow, G., Wilson, W., \& Auton, S. (1999). Health Level Seven Security Services Framework, HL7 Security Group.

Lee, S. Y. (2020). Cloud based Blockchain Technology for Personal Health, International Journal of Advanced Nursing Education and Research, 5(3), 14-20.

Lee, S. Y. (2021). Blockchain-based Medical Information Sharing Service Architecture. International Journal of IT-based Public Health Management, 8 (1).

Mallik, S. N. R., \& Neeraja, S. (2019). A SURVEY ON MAP REDUCE FRAMEWORK FOR CLUSTERING SECURITY. International Journal of Private Cloud Computing Environment and Management. 6(1).

National Electrical Manufacturers Association, (2015). National Electrical Manufacturers Association, Part 8: Network Communication Support for Message Exchange, 2015.

Pelgrum, W., Morton, Y., van Graas, F., Vikram, P., \& Peng, S. (2011). Multidomain analysis of the impact on natural and man-made ionosphere scintillations on GNSS signal propagation. Proc. ION GNSS, Portland, OR.

Peng, S., \& Morton, Y. (2010). A USRP2-Based Multi-Constellation and MultiFrequency GNSS Software Receiver for Ionosphere Scintillation Studies. Proc. ION ITM, San Diego, CA.

Rino, C. L. (2011). The Theory of Scintillation with Applications in Remote Sensing, Hoboken, NJ:IEEE and Wiley.

Sekhar, C., \& Suneetha, N. (2018). A Study on Data Categorization for Data Analytics. International Journal of Internet of Things and Big Data. 3(1), 1-6. 
Vikram, P. (2011). Event driven data collection system for studying ionosphere scintillation, MS thesis, Miami University, 2011

Xu, D., \& Morton, Y. T. (2018). A semi-open loop GNSS carrier tracking algorithm for monitoring strong equatorial scintillation. IEEE Transactions on Aerospace and Electronic Systems, 54(2), 722-738.

Zhang, P., Douglas C., White, J., \& Lenz, G. (2018). Chapter One - Blockchain Technology Use Cases in Healthcare. Advances in Computers, 111, 1-41. 